\title{
Hyperuricemia: a lifestyle change
}

\begin{abstract}
There has been a lot of recent excitement regarding elevated serum uric acid as it has been correlated with not just the traditional disorders associated with it, like gout and nephrolithiasis, but also with numerous other chronic diseases like chronic kidney disease, metabolic syndrome, hypertension, coronary artery disease etc which have emerged as the new epidemic of the twenty first century. Although numerous studies have explored the correlation of gout with these lifestyle disorders, none has as yet managed to elucidate the role, if any; hyperuricemia plays in the causation of these. The fact that the rise in mean serum uric acid levels parallels the increase in consumption of certain dietary products has also received a lot of attention. In recent times, light has been shed onto the exact mechanism of renal handling of uric acid, the transporters involved in the tubular excretion and reabsorption, which maintain uric acid homeostasis in our body. We know now that a slight disturbance can overwhelm this machinery and contribute greatly towards the development of hyperuricemia in a given individual. The purpose of this review is to provide a basic understanding into what position this molecule holds in the current clinical scenario and more importantly, how we got here.
\end{abstract}

Keywords: hyperuricemia, Sugar sweetened beverage, guanosine, LRP2 polymorphism
Volume 6 Issue 4 - 2018

\author{
Ramesh Aggarwal, Devika Dua, Sachin Kumar \\ Jain, Ritika Sud, Shiv Raj Meena \\ Department of Medicine, Lady Hardinge Medical College \& \\ Associated Hospitals, India
}

Correspondence: Dr. Ramesh Aggarwal, Associate Professor Department of Medicine, Lady Hardinge Medical College, New Delhi, India,Tel 98186266|4, Email rameshlhmc@gmail.com

Received: June 01, 2017 | Published: July 12, 2018

\section{Introduction}

Currently hyperuricemia is defined as serum uric acid level greater than $6.8 \mathrm{mg} / \mathrm{dL}$, this is a 'physico-chemical' definition, it is the solubility limit of uric acid in serum, beyond this level there is super saturation of bodily fluids and risk of precipitation. This rise in serum uric acid level started a few million years ago, when primates lost the gene for uricase enzyme, unlike the rest of the mammals who retained the ability to form allantoin and maintain serum uric acid levels in the range of $0.5-2 \mathrm{mg} / \mathrm{dL}$. Why this happened is not a question which can be answered with certainty but there are several interesting possibilities. Selection pressure could have led to the loss of uricase as elevated uric acid conferred upon the primates an evolutionary advantage. This advantage could be the antioxidant function of uric acid or the adjuvant properties of it, which enabled it to increase the immunogenicity of viral antigens. The most intriguing solution, first presented by Johnson et al., ${ }^{1}$ was that this rise in uric acid enabled the assumption of erect posture by the hominoids by preventing postural hypotension, a likely possibility given that the low salt diet prevalent at the time would predispose them towards it.

\section{Prevalence of Hyperuricemia}

Lets skip forward a few millennia; what was started by mother nature as, probably, an evolutionary mechanism has been taken further by the lifestyle choices of human beings over the past few decades with unfortunate, pathological consequences. Since our body now has limited capacity for uric acid excretion any increased load can predispose us towards the development of hyperuricemia. In this regard, our lifestyle choices, especially dietary intake, have received a lot of attention as a probable culprit. The prevalence of hyperuricemia has steadily increased over the past few decades. A surveillance study, NHANES III, undertaken from 1990-1999, to identify 10-year trends in gout and/ or hyperuricemia found that they increased in population by 2 per 1000 person years with maximal increase in the over 65 year age group where prevalence increased from $31 / 1000$ person years to $41 / 1000$ person years. ${ }^{2}$ NHANES $2007-2008$ found an increase in mean serum rate level of population by $0.15 \mathrm{mg} / \mathrm{dL}$ with maximal increase in men and an increase in the prevalence of hyperuricemia by $3.2 \%$ in comparison with the NHANES III. $^{3}$ These two taken together demonstrate the consistent rise in mean uric acid levels and prevalence of hyperuricemia over the past two decades. The overall prevalence of hyperuricemia in adult population was found to be $21.6 \% .^{3}$ The prevalence of hyperuricemia in adult Japanese men is estimated to be around $30 \%$ (Table 1). ${ }^{4}$ These figures highlight how deeply entrenched this problem is.

Table I Prevalence of hyperuricemia

\begin{tabular}{ll}
\hline Country/ Region & Prevalence \\
\hline U.S.A & $2.7 \%(1987-94) / 3.9 \%(2007-08)$ \\
Japan(Males) & $15-16 \%(1983) / 30 \%(2003)$ \\
Mainland China & $13.3 \%(2000-2013)$ \\
SEAR & $9-25 \%(2015)$ \\
Western Europe & $9-16 \%$ \\
\hline
\end{tabular}

\section{Hyperuricemia: Beverages, diet and genes}

The association between alcohol and hyperuricemia is well known. The type and amount both affect the serum uric acid level. A recent study showed that prevalence of hyperuricemia increases proportionally with alcohol consumption in the male drinkers with an approximately 1.7 -fold higher risk of hyperuricemia in heavy drinkers as compared with non-drinkers. ${ }^{5}$ Interestingly prevalence rates did not differ much among drinkers and non drinkers in the female population. NHANES III revealed that uric acid levels increased with beer or liquor intake but not with wine, with maximal increase noted with beer consumption. ${ }^{6}$ The high guanosine content of beer is likely responsible for this increase in uric acid levels. 
Other dietary factors have also received a lot of attention as being potentially hyperuricemic. The third NHANES was a rich source of information and it was found that serum uric acid increased by a mean of $0.48 \mathrm{mg} / \mathrm{dL}$ in the highest quintile of meat intake, and by 0.16 $\mathrm{mg} / \mathrm{dL}$ in the highest quintile of seafood intake. ${ }^{7}$ Total protein intake was not independently related with hyperuricemia and surprisingly neither was purine rich vegetable intake (peas, mushroom, lentils, spinach), both of these dietary practices were traditionally believed to be contributory. ${ }^{8}$ Low fat dairy, specifically milk and yogurt, were found to be negatively associated with uric acid levels, with difference approaching $-0.21 \mathrm{mg} / \mathrm{dL}$ for total dairy intake. ${ }^{7}$

Sugar sweetened beverage (SSB) consumption is associated with increased serum uric acid level, and the increase is quantity dependant with an increase in mean urate level by $0.42 \mathrm{mg} / \mathrm{dL}$ observed in those who consumed 4 or more servings of SSB per day. ${ }^{9}$ Fructose, specifically as a part of the high fructose corn syrup, used as an industrial sweetener in these SSBs has emerged as a major contributory factor towards hyperuricemia, with many blaming it for the increased prevalence over past two decades. However another study found that though SSB consumption is positively associated with hyperuricemia, energy adjusted fructose intake is, independently, not associated with urate levels. ${ }^{8}$

A recent area of study has been the interaction of SSB intake and specific genetic variations with the interaction accounting for increased risk of developing hyperuricemia.The basic underlying mechanism seem to be fractional under excretion of urate by the kidneys. SLC2A9 is a urate transporter, which exchanges uric acid for glucose and fructose, and is associated with resorption of urate in the proximal tubule. Loss of function mutations have been found in certain studies to be protective for hyperuricemia and gout. ${ }^{10} \mathrm{~A}$ recent study showed that urate transport via SLC2A9 is physiologically influenced by intake of SSB. Increased SSB intake ameliorates the effect of the gout protective allele of SLC2A9. ${ }^{11}$ This exemplifies the gene-environment interaction in a complex polygenic, multifactorial disorder, highlighting the importance of lifestyle modification especially in those populations which are inherently susceptible for hyperuricemia.

Another consistently important association has been found between uric acid and body bulk of an individual, independent of their dietary intake. Body weight, height, BMI, BSA are all powerful predictors of the serum urate levels of an individual. ${ }^{12-14}$ Another interesting recent study found that the effect of BMI on SUA was modified by LRP2 polymorphism in Japanese adults. The effect of BMI was significantly greater in males with $\mathrm{CC} / \mathrm{CT}$ genotypes as compared to those with the TT genotype. In females, however, the strongest effect of BMI was associated with the TT genotype. ${ }^{15}$

Lead intoxication also leads to hyperuricemia and gout. This association was highlighted in the era of prohibition in the USA when 'moonshine', illegal homemade liquor was brewed in lead lined vessels. In the contemporary context this effect has been noted in populations living in an area with high levels of lead pollution. In one such study conducted in China, blood lead levels correlated positively with serum uric acid levels. ${ }^{16}$ Among other environmental toxins, cadmium levels also found to be associated with increased mean serum rate levels. ${ }^{17}$

Keeping all the current available knowledge in mind the ACR issued specific guidelines with recommendations for lifestyle modification in hyperuricemia. ${ }^{18}$ They recommend avoiding organ meats, high fructose corn syrup sweetened beverages and alcohol overuse (defined as more than 2 servings per day for males and more than 1 serving per day for females.) They suggest limitation of red meat and seafood intake, naturally sweetened fruit juice, table sugar, salt and alcohol. They also advise increased intake of low fat or nanfat dairy products and vegetables.

\section{Conclusion}

Serum uric acid is a mirror reflecting the many lifestyle choices we make with regard to our diet and alcohol consumption. There is a section of the population which might be especially vulnerable to developing hyperuricemia owing to their genetic predisposition. We have greater understanding today of the impact renal under excretion has on serum uric acid levels, though further advancements are needed to fully elucidate the impact of each individual genetic variation.

Knowledge of these factors, however, is just like a stone thrown into a deep pond, it creates a ripple but doesn't really displace the water. The actual changes we have to make in our diet and other lifestyle choices remain a challenge, a very difficult one but hopefully not an insurmountable one.

\section{Acknowledgements}

None.

\section{Conflict of interest}

Author declares there is no conflict of interest in publishing the article.

\section{References}

1. Johnson RJ, Andrews P, Benner SA, et al. Woodward award. The evolution of obesity: insights from the mid Miocene. Trans Am Clin Climatol Assoc. 2010;121:295-305

2. Wallace KL, Riedel AA, Joseph-Ridge N, et al. Increasing prevalence of gout and hyperuricemia over 10 years among older adults in a managed care population. J Rheumatol. 2004;31(8):1582-1587.

3. Zhu Y, Pandya BJ, Choi HK. Prevalence of gout and hyperuricemia in the US general population: the National Health and Nutrition Examination Survey 2007-2008. Arthritis Rheum. 2011;63(10):3136-3134.

4. Hakoda M. Recent trends in hyperuricemia and gout in Japan. Japan Med Assoc J. 2012;55(4):319-323.

5. Li Z, Guo X, Liu Y, et al. The relation of moderate alcohol consumption to hyperuricemia in a rural general population. Int J Environ Res Public Health. 2016;13(7):732.

6. Choi HK, Curhan G. Beer, liquor, and wine consumption and serum uric acid level: the Third National Health and Nutrition Examination Survey. Arthritis Rheum. 2004;51(6):1023-1029.

7. Choi HK, Liu S, Curhan G. Intake of purine-rich foods, protein, and dairy products and relationship to serum levels of uric acid: the Third National Health and Nutrition Examination Survey. Arthritis Rheum. 2005;52(1):283-289.

8. Zgaga L, Theodoratou E, Kyle J, et al. The association of dietary intake of purine rich vegetables, sugar-sweetened beverages and dairy with plasma rate, in a cross sectional study. PLoS One. 2012;7(6):e38123.

9. Choi JW, Ford ES, Gao X, et al. Sugar sweetened soft drinks, diet soft drinks, and serum uric acid level: the Third National Health and Nutrition Examination Survey. Arthritis Rheum. 2008;59(1):109-116. 
10. Voruganti VS, Laston S, Haack K et al. Serum uric acid concentrations and SLC2A9 genetic variation in Hispanic children: the Viva La Familia Study. Am J coin Nutr. 2015;101(4):725-732.

11. Batt C, Phipps Green AJ, Black MA, et al. Sugar sweetened beverage consumption: a risk factor for prevalent gout with SLC2A9 genotype-specific effects on serum urate and risk of gout. ANN Rheum Dis. 2014;73(12):2101-2106.

12. Tanaka K, Ogata S, Tanaka H, et al. The relationship between body mass index and uric acid: a study on Japanese adult twins. Environ Health Prev Med. 2015;20(5):347-353.

13. Juraschek SP, Miller ER, Gelber AC. Body mass index, obesity, and prevalent gout in the United States in 1988-1994 and 2007-2010. Arthritis Care Res. 2013;65(1):127-132.
14. Gout risk linked to BMI. Jody A Charnow, editor. Nephrology cover articles, Renal and Urology News. 2014

15. Suma S, Naito M, Okada R, et al. Association between body mass index and serum uric acid levels in a Japanese population were significantly modified by LRP2 rs2544390. Nayoga J of Med Sci. 2014;76(3-4):333-339.

16. Dai H, Huang Z, Deng Q, et al. The effects of lead exposure on serum uric acid and hyperuricemia in Chinese adults: a cross sectional study. Int $J$ Environ Res Public Health. 2015;12(8):9672-9682.

17. Honglin Sun, Ningjian Wang, Chi Chen, et al. Cadmium exposure and its association with serum uric acid and hyperuricemia. Sri Rep. 2017;7:550.

18. Khanna d, Fitzgerald JP, Khanna PP, et al. Specific recommendation on general health, diet, lifestyle measures for patients with gout. Arthritis Care Res (Hoboken). 2012;64(10):1431-1446. 